\title{
OPTICAL NUCLEAR ACTIVITY IN RADIO GALAXIES IN CLUSTERS
}

\author{
H. K. C. Yee \\ Département de Physique, Université de Montréal \\ Montréal, PQ, H3C 3J7, Canada \\ M. M. De Robertis \\ Department of Physics, York University \\ North York, ON, M3J 1P3, Canada
}

\section{Introduction}

It has traditionally been accepted that quasars and strong emission-line radio galaxies, although situated in regions of enhanced galaxy density, are rarely found within rich clusters (Yee and Green 1984; Longair and Seldner 1978). The recent finding by Yee and Green (1987) that at $z \gtrsim 0.5$ a substantial fraction of optically bright radio-loud quasars are found in galaxy clusters as rich as Abell class 1, suggests that a substantial evolution of the environments of rich clusters has taken place over this short time period. Similar results for radio galaxies have also been obtained by Hill and Lilly (1988, private communication). If this is an evolutionary effect, then at least some first-rank elliptical galaxies in low-redshift rich clusters must contain remnants of active galactic nuclei (AGN). Motivated by this, we attempted to find traces of AGN-like activity in a sample of FR class I radio galaxies which are known to be "quiescent" absorption-line systems situated in low-redshift clusters.

\section{Observations and Results}

We have obtained high signal-to-noise ratio, long-slit CCD spectra at the CFHT for a sample of low-redshift 3CR radio galaxies $(z \leq 0.05)$ situated in moderately rich environments. We observed 6 radio galaxies and 5 bright cluster ellipticals as a comparison sample using a slit width of $1^{\prime \prime}$ covering the rest wavelength region $4500-7100 \AA$ at $5 \AA$ resolution.

Even before removing the underlying absorption-line spectrum, it is apparent that all 6 $3 \mathrm{CR}$ radio galaxies have substantial nuclear emission- usually $\mathrm{H} \alpha$, [N II] $\lambda \lambda 6548,6583$, and often [S II] $\lambda \lambda 6716,6731$ and [O I] $\lambda 6300,6363$. Subtracting the absorption-line component of each spectrum using absorption-line template galaxies or the galaxy light outside the nucleus, a "nuclear spectrum" for each object was derived using an optimal extraction technique. The emission is spatially unresolved in each case, while the emission-line profiles are resolved with widths and intensity ratios similar to that found in AGNs rather than $H$ II region galaxies. Some spectra show evidence for underlying weak, broad $\mathrm{H} \alpha$ emission. An example is shown in Figure 1. The typical $\mathrm{H} \alpha$ luminosity for these galaxies is $\sim 10^{41} \mathrm{erg} / \mathrm{s}$. This is to be compared with the $\mathrm{H} \alpha$ luminosities $\$ 10^{40} \mathrm{erg} / \mathrm{s}$ for dwarf Seyferts (Filippenko 1988) and $\sim 10^{42} \mathrm{erg} / \mathrm{s}$ for classical Seyfert 2 galaxies (based on Dahari and De Robertis 1988). Furthermore, at least 5 of the 6 radio galaxies have companions at similar redshifts (within a projected separation of 457 
$15 \mathrm{kpc}$ ), and most of these are emission-line galaxies in their own right. In comparison, all 5 bright ellipticals have weak, narrow emission lines similar to H II region galaxies (see Fig. 1), and some have spatially extended emission.

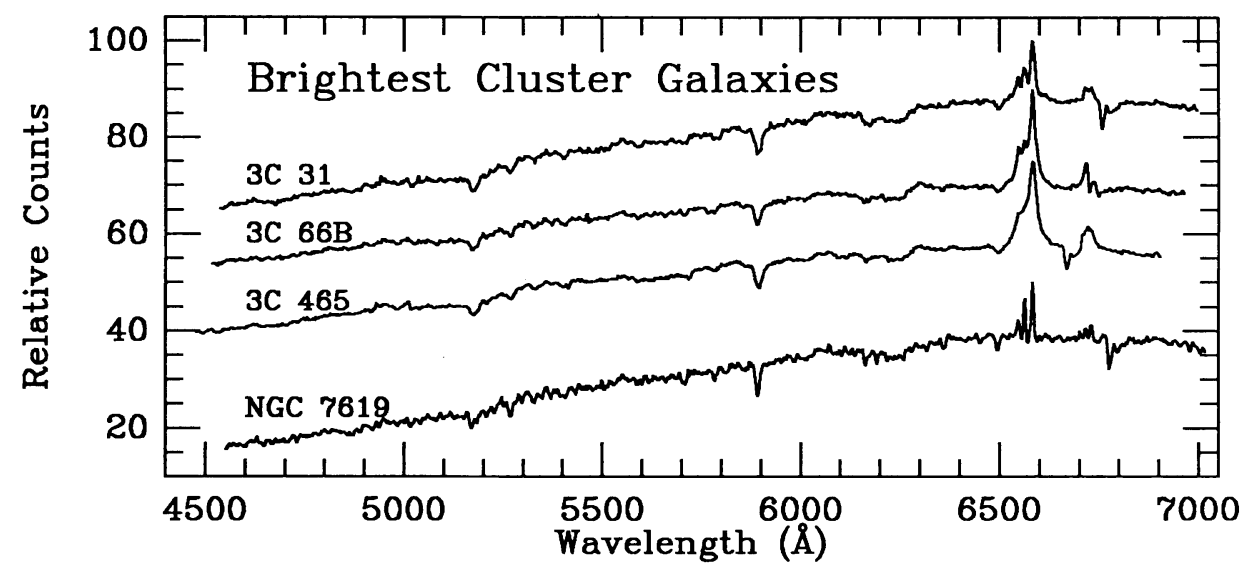

Figure 1. Examples of nuclear spectra of radio galaxies (top 3) and a control sample galaxy (bottom) in the rest frame. Note the differences in the $\mathrm{H} \alpha+[\mathrm{N} \mathrm{II}]$ region.

\section{Discussion}

It appears that there are two very distinct bright cluster member populations which can be distinguished by their radio properties. These preliminary results, along with the findings of Yee and Green that bright radio-loud quasars in rich clusters may have dimmed substantially between the redshifts of 0.6 and 0.4 , are consistent with the scenario that low-redshift optically "quiescent" radio galaxies in clusters may be the remnants of more active objects, ranging from broad-line radio galaxies to $M \approx-30$ radio-loud quasars that were once situated in the centers of rich clusters. These "remnants" are either still active at an extremely low level, or they are experiencing a minor rekindling of nuclear activity due to the perturbation of a close companion.

Assuming a connection between these two populations, the subsidence of optical activity may have the same origin as the change in the radio structure. Two possible mechanisms can be used to explain this evolution. One is a decrease in the frequency of effective galaxy interactions in the cores of rich clusters as they virialize. The other is an increase in the density of the intra-cluster medium resulting from stripping which effectively starves the central engine in the nucleus, and at the same time, causes the radio morphology to become more diffuse. It is likely that both mechanisms play a role in quasar evolution.

\section{References}

Dahari, O., and De Robertis, M.M. 1988, Ap. J. Suppl., 67, 249.

Filippenko, A.V. 1988, in Proceedings of the third George Mason Astrophysics Workshop on Supermassive Black Holes, ed. M. Kafatos (Cambridge: Cambridge Univ. Press) p. 104.

Longair, M.S., and Seldner, M. 1979, M.N.R.A.S. 189, 433.

Osterbrock, D.E., Koski, A.T., and Phillips, M.M. 1976, Ap. J., 206, 898.

Yee, H.K.C., and Green, R.F. 1984, Ap. J., 280, 79.

Yee, H.K.C., and Green, R.F. 1987, Ap. J., 319, 28. 


\section{DISCUSSION}

MARSHALL Did you find any differences in the colors or luminosities of the central source in the nearby $3 \mathrm{C}$ galaxies compared to the distant FR II galaxies?

YEE It is extremely difficult to actually measure the central continuum source because it is very weak compared to the host galaxy nucleus. If we assume that the luminosity of the central source is proportional to $\mathrm{H} \alpha$ flux, then these objects are typically 10 to 100 times fainter than the more distance FR II nuclei.

BLANDFORD Barthel has advocated the view that Type II radio galaxies are obscured radio quasars. A consequence is that galaxies and quasars of comparable extended radio properties should have comparable narrow-line luminosities. Do your data allow you to comment on this?

YEE No, since our data concern Type I morphology galaxies. However, I find the model you mention a little bit difficult to accept since it would require 1) a strong inverse dependence of the strength of narrow lines on the luminosity of the continuum, (there is some evidence that, in AGNs, the luminosity of [O III] $\lambda 4959+\lambda 5007$ is not proportional to that of $\mathrm{H} \beta$ ); and 2) the strength of narrow lines to be a function of the colors of the continuum. 